Check for updates

Cite this: RSC Adv., 2017, 7, 52125

\section{In situ second-harmonic generation mediated photodynamic therapy by micelles co- encapsulating coordination nanoparticle and photosensitizer $\uparrow$}

\author{
Xuyang Zhou, ${ }^{a}$ Yang Chen, ${ }^{a}$ Jian Su, (DD ${ }^{b}$ Xiaohe Tian, (D) ${ }^{c}$ Yonghuang Luo*a \\ and Lei Luo (D)*a
}

\begin{abstract}
The indirect excitation of a photosensitizer mediated with nonlinear optical mechanism is a strategy for applying tissue penetration enhanced near infrared (NIR) light on photodynamic therapy (PDT). Herein, we introduced a NIR-PDT strategy that employed a nonlinear optical nano-conveyor named AHU-1 bearing strong second-harmonic generation (SHG) properties. AHU-1 could emit exactly half of the incident wavelengths for indirectly exciting the strong absorption soret band of the photosensitizers. Biocompatible micelles (mPEG-PDLLA) were applied to simultaneously encapsulate AHU-1 and photosensitizer, chlorin e6 (Ce6), for increasing their solubility and stability. The size and morphology of cargo loaded micelles were characterized by DLS and TEM. The cytotoxicity studies, live cell imaging and in situ photodynamic efficacy were conducted on B16 melanoma cells, and the SHG-mediated PDT effectively eliminated the cancer cells. This study provides a novel strategy for the application of

nonlinear optical materials in photodynamic therapy.
\end{abstract}

Received 3rd July 2017

Accepted 28th October 2017

DOI: 10.1039/c7ra07334h

rsc.li/rsc-advances

\section{Introduction}

Photodynamic therapy (PDT) is able to non-invasively treat cancer by combining light, oxygen and photosensitizers (PSs). ${ }^{1}$ However, the application of PDT is largely hindered by limited light penetration, ${ }^{2}$ systemic toxicity derived from off-site localization and poor water solubility of PSs. ${ }^{3}$

In order to address the problem of limited light penetration, near-infrared light (700-1100 nm) is used in PDT because of its deeper tissue penetration and minimal damage to healthy tissues. ${ }^{4}$ To combine near-infrared light with PDT perfectly, various PSs activation strategies are under investigation. These strategies are mainly classified into two categories: the direct NIR-excitation of $\mathrm{PSs}^{5,6}$ and the indirect NIR-excitation of PSs via nonlinear optical conveyors. ${ }^{7}$ For NIR-excitable PSs, a large twophoto absorption (TPA) cross-section is indispensable. However, most PSs, particularly of the porphyrin family, possess an inadequate TPA cross-section (1-10 GM), which limits the

${ }^{a}$ College of Pharmaceutical Sciences, Southwest University, Chongqing 400715, P. R. China. E-mail: luoyonghuang@126.com; Drluolei@swu.edu.cn; Tel: +86 02368251225

${ }^{b}$ State Key Laboratory of Coordination Chemistry, School of Chemistry and Chemical Engineering, Nanjing University, Nanjing 210093, P. R. China

${ }^{c}$ Department of Chemistry, Key Laboratory of Functional Inorganic Material Chemistry of Anhui Province, Anhui University, Hefei 230039, P. R. China

$\dagger$ Electronic supplementary information (ESI) available. See DOI: $10.1039 / \mathrm{c} 7 \mathrm{ra07334h}$
NIR-excitation of PSs. ${ }^{8}$ Therefore, it is widely used to modify the chemical structure of PSs or to couple them to other chromophores with high TPA cross-section, aiming at increasing the probability of NIR-excitation because of the difficulties in the synthesis of NIR-excitable PSs. However, these modifications may result in complex, hardly predictable changes in the pharmacokinetics of PDT drugs. ${ }^{\mathbf{9}, 10}$ Unlike the former strategy, the indirect NIR-excitation of PSs shows more flexibility in the choice of PSs because of the application of nonlinear optical conveyors such as up conversion nanoparticles (UCNPs) and two-photon absorption materials. ${ }^{11,12}$ However, their upconverted spectra are fixed since synthesis, which leads to insufficient overlapping with the spectra of acceptors (PSs), and the fluorescence resonance energy transfer (FRET) efficiency is not ideal. $^{13}$ Furthermore, their potential long-term safety and potential photodamage induced by NIR laser are still under investigation.

SHG is a second-order nonlinear optical process in which two photons at frequency $\omega$ interacting with noncentrosymmetrical media combine to form a new photon with twice the energy, and therefore double the frequency $(2 \omega)$ and half the wavelength of the initial photons. ${ }^{14}$ SHG materials have made considerable progress in ion sensors, ${ }^{\mathbf{1 5}}$ biological imaging ${ }^{16,17}$ and $\mathrm{PDT}^{18-20}$ owing to the advantages of strong penetration ability, high resolved localization and low phototoxicity. Working as a nonlinear optical conveyor, SHG material is able to halve the excitation wavelength to match the 


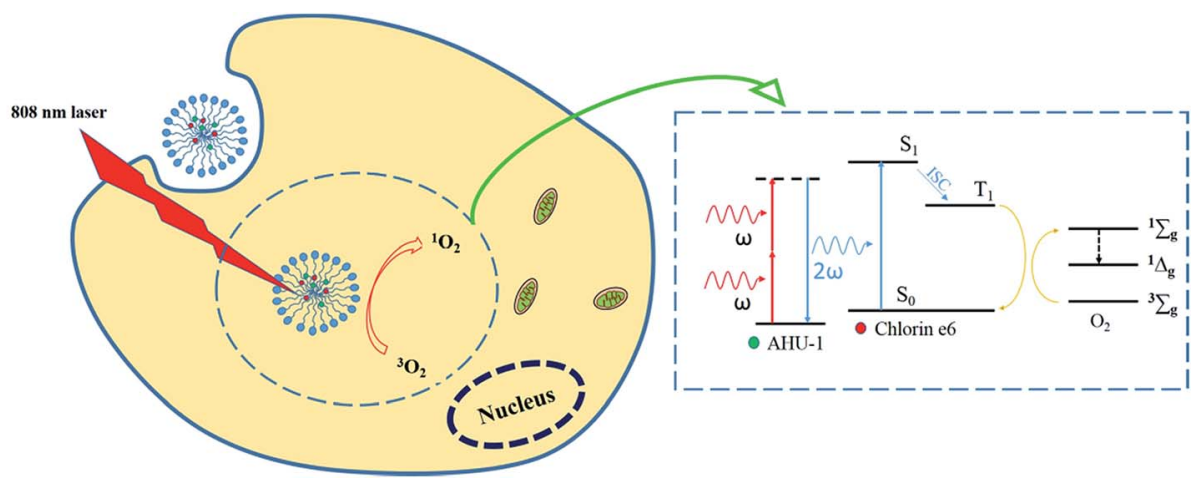

Scheme 1 Schematic of polymer nanoscale-based SHG PDT.

maximum one-photon absorption of any PS. In addition, compared to the two-photon conveyor, SHG materials do not require the formation of excited states, which could substantially eliminate photobleaching and phototoxicity. In our recent study, a biodegradable nanoscaled coordination polymer (AHU-1) with labile metal ligand bonds was synthetized, and possessed high SHG signals. ${ }^{21}$ However, AHU-1 is insoluble and dissociates in water, which disables its SHG function. In order to enable SHG with PDT, a nano polymeric vehicle, $\mathrm{mPEG}_{2000^{-}}$ PDLLA $_{2000}$ copolymer micelles, ${ }^{22}$ was used to simultaneously encapsulate AHU-1 and Ce6 (Ce6/AHU-1 co-encapsulated micelles, CAM). The application of polymer micelles aims to make up for the poor water-stability of the contents and codelivers them into cancer cells for effective PDT. After the micelles are taken up by cancer cells, AHU- 1 can upconvert NIR laser to visible light in situ through the SHG mechanism for triggering PDT. The concept of this work is illustrated in Scheme 1.

In this work, the in vitro characterizations of AHU-1 and CAM were conducted for investigating stability. Then, the live cell biological imaging of CAM was carried out to study its cellular uptake and the stability of SHG signals from AHU-1 in the cell. Moreover, the dark cytotoxicity and PDT effect of CAM in the cell were evaluated by MTT assay and confocal laser scanning microscopy to confirm the PDT efficacy based on SHG mechanism. Thus, our work presented NIR laser-excited micelle formulation for PDT in cancer cells, in which a strong penetration depth and high-efficacy PDT could be realized.

\section{Experimental}

\subsection{Materials}

Chlorin e6 (Ce6, $\left.M_{\mathrm{W}}=596.68\right)$ was purchased from Thermo Fisher Scientific. Methoxy polyethylene glycol-polylactide $\left(\mathrm{mPEG}_{2000}-\mathrm{PDLLA}_{2000}\right)$ was purchased from Shanghai Liang Chemical Co. Ltd., and 9,10-anthracene-dipropionic acid disodium salt (ADPA) was purchased from Santa Cruz Biotechnology. Fetal bovine serum (FBS) was purchased from Hangzhou Sijiqing Biological Co., Ltd., and 3-(4,5-dimethylthiazol-2-yl)-2,5-diphenyltetrazolium bromide (MTT) was produced by Sigma-Aldrich. PRMI-1640 medium was supplied by Nanjing KeyGEN Biotechnology Corp., Ltd.
Methanol, chloroform and dimethyl sulfoxide were purchased from Sigma-Aldrich.

\subsection{Synthesis of tpatpy}

The tpatpy ligand was prepared as described in our recent literature ${ }^{19}$ It was synthesized via the Vilsmeier-Haack reaction with 4-(diphenylamino) benzaldehyde as the raw material. Furthermore, 4-acetylpyridine $(2.67 \mathrm{~g}, 22.00 \mathrm{mmol})$ was added to a solution of 4 -formyltriphenylamine $(2.73 \mathrm{~g}, 10.00 \mathrm{mmol})$ in EtOH $(50 \mathrm{~mL})$. Then, $5 \mathrm{~mL}$ cooled KOH $(1.71 \mathrm{~g}, 30.00 \mathrm{mmol})$ aqueous solution was then added, followed by aqueous $\mathrm{NH}_{3}$ $(25 \%, 30 \mathrm{~mL})$, and the resulting solution was stirred at $85^{\circ} \mathrm{C}$ for $24 \mathrm{~h}$.

\subsection{Synthesis of nano-scale AHU-1}

AHU-1: $\left[\mathbf{Z n}(\text { tpatpy }) \mathbf{C l}_{2} \cdot\left(\mathbf{H}_{2} \mathbf{O}\right)\right]_{n}$. The nano-scale AHU-1 was synthesized in a $10 \mathrm{~mL}$ centrifuge tube with ultrasonic cleaners. In ultrasonic environment, $4 \mathrm{~mL} 5 \times 10^{-4} \mathrm{~mol} \mathrm{~L}^{-1} \mathrm{ZnCl}_{2}$ solution was added dropwise into $4 \mathrm{~mL} 5 \times 10^{-4} \mathrm{~mol} \mathrm{~L}^{-1}$ tpatpy solution, stirred intensely for 10 minutes, and then kept standing for several minutes. The liquid supernatant was stored for further use.

\subsection{Preparations of copolymer micelles}

Blank micelles (BM), Ce6-encapsulated micelles (CM), AHU-1encapsulated micelles (AM), Ce6/tpatpy co-encapsulated micelles (tCM) and Ce6/AHU-1 co-encapsulated micelles (CAM) were prepared using the thin-film hydration method. ${ }^{23}$ Moreover, $20 \mathrm{mg}$ mPEG $_{2000}-$ PDLLA $_{2000}$ was dissolved in $3 \mathrm{~mL}$ methanol. Then, the polymer solution was mixed with $2.6 \mathrm{~mL}$ AHU-1 solution $\left(0.15 \mathrm{mg} \mathrm{mL}{ }^{-1}\right)$ and/or $1 \mathrm{~mL} \mathrm{Ce} 6$ methanol solution $\left(0.2 \mathrm{mg} \mathrm{mL}^{-1}\right)$. The mixed solution was then evaporated in a $50 \mathrm{~mL}$ rotary evaporation bottle at $40{ }^{\circ} \mathrm{C}(100 \mathrm{rpm})$ under vacuum to form a drug-loaded polymer film. Then, the thin-film was hydrated by adding $2 \mathrm{~mL} 0.01 \mathrm{M}$ phosphate buffer saline (PBS, pH 7.4) and stirred at room temperature for 6 hours to form micelles, after which the solution was filtered through a $0.22 \mu \mathrm{m}$ membrane filter to remove unloaded hydrophobic drugs. The final product was stored at $4{ }^{\circ} \mathrm{C}$ for further use. BM, $\mathrm{CM}$, and AM were prepared using the same method as described 
above. In order to eliminate the possibility of Ce6 and AHU-1 molecule forming nanocrystals in aqueous solution through the membrane, the same protocol without polymer was used with Ce6 and AHU-1. The final solution was filtered through a $0.22 \mu \mathrm{m}$ membrane, and its absorption curve was determined using a UV-vis spectrophotometer (see ESI†).

\subsection{Characterization of micelles and nanoscaled AHU-1}

Transmission electron microscopy (TEM) images were obtained on a JEM-1200EX transmission electron microscope operating at $120 \mathrm{kV}$. Samples were prepared by placing a drop of diluted CAM dispersion or AHU-1 solution on a carbon-coated copper grid (150 mesh) and drying for $10 \mathrm{~min}$ in air. The specimens were not stained. The size and zeta potentials of the micelles were measured with a Zeta-sizer (Malvern Instruments, Malvern, UK) at a wavelength of $633 \mathrm{~nm}$ at $25^{\circ} \mathrm{C}$. All samples were measured at a scattering angle of $173^{\circ}$ (backscatter) using the 'General purpose' analysis model and the default size analysis parameters as well as a refractive index of 1.59 for the polystyrene particle matrix as a sample parameter. Each sample was measured 13 runs per measurement and 15 seconds per run. Distinct fractions of the runs were removed from the final measurement calculation using a dust filtration algorithm. The intensity-weighted harmonic mean particle diameter (Zaverage) and the polydispersity index (PI) according to ISO 22412 were used to compare sizes and size distributions of the different samples. Samples with good 'Expert Advice' results were satisfactory for experimental use. Each measurement was performed in triplicate. UV-vis spectra were recorded using a UV-6100 spectrophotometer (Shanghai Mapada Instruments Co., Ltd, China). The stability of micelles in a RPMI-1640 cell culture medium with $10 \%(\mathrm{v} / \mathrm{v})$ fetal bovine serum was simply investigated through measurement of the particle size at different time points under $37^{\circ} \mathrm{C}$.

The in vitro release behaviors of AHU- 1 and $\mathrm{Ce} 6$ from CAM were assessed using a dialysis method in PBS at $37^{\circ} \mathrm{C}(\mathrm{pH} 6.5$ and $\mathrm{pH} 7.4$, respectively). Furthermore, $5 \mathrm{~mL}$ of CAM solution at a drug concentration of $1 \mathrm{mg} \mathrm{mL}^{-1}$ was placed in a dialysis bag (molecular weight cut-off $=3500 \mathrm{Da}$ ), and the bag was immersed in $100 \mathrm{~mL}$ of $0.01 \mathrm{M}$ PBS with stirring at $37^{\circ} \mathrm{C}$ for $36 \mathrm{~h}$. An aliquot was withdrawn at set time points and passed through a $0.22 \mu \mathrm{m}$ membrane filter before quantification. The cumulative release of AHU- 1 and Ce6 was calculated based on the absorbance at the corresponding maximum absorption wavelengths.

\subsection{Singlet oxygen $\left({ }^{1} \mathrm{O}_{2}\right)$ detection}

In this study, the amount of singlet oxygen was detected by a singlet oxygen sensor named 9,10-anthracene-dipropionic acid disodium salt (ADPA), because the newly generated singlet oxygen could cause an absorbance decrease of the chemical probe at around $400 \mathrm{~nm}$. Briefly, $1.5 \mathrm{~mL}$ of micelles solution was mixed with $7.5 \mu \mathrm{L}$ of ADPA solution $(16 \mathrm{mM})$ and then exposed to laser excitation at $808 \mathrm{~nm}$ for $30 \mathrm{~min}(808 \mathrm{~nm}$ laser beam was purchased from Xian Midriver Optoelectronics Technology Co., Ltd, China.). The absorbance of the probe was measured at the same time-scale to evaluate the generation of singlet oxygen in different samples.

\subsection{Cell culture}

B16 melanoma cells were supplied by KeyGEN Ltd. B16 melanoma cells were cultured in a humidified incubator at $37{ }^{\circ} \mathrm{C}\left(5 \% \mathrm{CO}_{2} /\right.$ 95\% air atmosphere) with RPMI-1640 medium, supplemented with $10 \%(\mathrm{v} / \mathrm{v})$ fetal bovine serum (FBS). The cells were harvested with $0.02 \%$ EDTA and $0.025 \%$ trypsin and rinsed. The resulting cell suspension was used for the following experiments.

\subsection{Dark cytotoxicity assay of samples}

The dark cytotoxicity of free tpatpy (in DMSO), free Ce6 (in DMSO), BM, AM, CM and CAM was tested using the standard MTT assay. B16 melanoma cells were seeded into a 96-well plate at a density of $1.0 \times 10^{4}$ cells per well in RPMI-1640 medium containing $10 \%$ FBS for $24 \mathrm{~h}$ at $37^{\circ} \mathrm{C}$. After cell attachment, the complete medium was replaced by the incomplete medium containing different concentrations of micelle samples and incubated for $24 \mathrm{~h}$ (free tpatpy and free Ce6 were diluted with PBS and subsequently added into fresh incomplete medium to reach the target concentration). After that, the medium was replaced with fresh medium, $10 \mu \mathrm{L}$ of $5 \mathrm{mg} \mathrm{mL}{ }^{-1}$ MTT solution was added to each well, and the cells were incubated at $37{ }^{\circ} \mathrm{C}$ for another $4 \mathrm{~h}$. Then, the medium was removed and $150 \mu \mathrm{L}$ DMSO was added. A microplate reader was used to record the absorbance of each well at $570 \mathrm{~nm}$, which can be used to calculate the cell viabilities compared to those of the control untreated cells (100\% survival).

\subsection{Cellular uptake imaging}

The confocal laser scanning microscope (CLSM) ZEISS 710 was used to study the cellular uptake of the micelles. B16 melanoma cells at a density of $5 \times 10^{5}$ per hole were seeded in 6-well plates and incubated at $37^{\circ} \mathrm{C}$ in $5 \%$ of $\mathrm{CO}_{2}$ for $24 \mathrm{~h}$. Then, the culture medium was replaced with fresh culture medium, which contained CAM $\left(2 \mu \mathrm{g} \mathrm{mL}{ }^{-1}\right.$ Ce6, $\left.4 \mu \mathrm{g} \mathrm{mL}{ }^{-1} \mathrm{AHU}-1\right)$ and AM ( $4 \mu \mathrm{g} \mathrm{mL} \mathrm{m}^{-1}$ AHU-1), respectively. After incubation for another $2 \mathrm{~h}$, the cells were washed three times with PBS to remove the free micelles. Finally, the two-photon images of CAM and AM were recorded with an incident wavelength of $760 \mathrm{~nm}$. The SHG images of CAM and AM were obtained with an incident wavelength of $980 \mathrm{~nm}$. The single-photon images of CAM and AM were recorded with the incident wavelength of $610 \mathrm{~nm}$.

\subsection{In vitro PDT efficacy}

The in vitro PDT efficacy of CAM was evaluated by the standard MTT assay and observed under a confocal laser scanning microscope. In brief, B16 melanoma cells were seeded into 96-well plates and incubated with CAM $\left(6 \mu \mathrm{g} \mathrm{mL}{ }^{-1}\right.$ AHU-1 and $3 \mu \mathrm{g} \mathrm{mL} \mathrm{m}^{-1}$ Ce6) for $24 \mathrm{~h}$. After incubation, the cells were exposed to $808 \mathrm{~nm}$ NIR light $\left(0.3 \mathrm{~W} \mathrm{~cm}^{-2}\right)$ for $0 \mathrm{~min}, 5 \mathrm{~min}$ and $10 \mathrm{~min}$. The controls were the blank cells treated with the same light dosage. Then, $10 \mu \mathrm{L}$ of $5 \mathrm{mg} \mathrm{mL}^{-1}$ MTT solution was added to the wells, and the cells were incubated for another $4 \mathrm{~h}$. Finally, the medium was replaced by $150 \mu \mathrm{L}$ DMSO to dissolve the formazan crystals 
generated by living cells. The absorbance at a wavelength of $570 \mathrm{~nm}$ was used to evaluate the photodynamic therapy effect of CAM in vitro. In addition, the changes in cell morphology after PDT were also investigated using the confocal laser scanning microscope.

\section{Results and discussion}

\subsection{Characterization of tpatpy and AHU-1}

The specific characterization data of tpatpy and AHU-1 were provided in our former research. ${ }^{19}$

\subsection{Characterization of micelles}

Owing to the relatively low water-stability of nanoscaled AHU-1 and $\mathrm{Ce} 6, \mathrm{mPEG}_{2000}-\mathrm{PDLLA}_{2000}$ diblock copolymer was used as a 'raincoat' and delivery vehicle to protect both the hydrophobic matters from water and ensure colloidal stability in water. The polymer was prepared into micelles to encapsulate nano-AHU-1 or Ce6. As shown in Fig. 1a, the prepared AM, CM and CAM were faint yellow, gray and pale yellow, respectively. It confirmed initially the co-loading of nano-AHU-1 and Ce6 in CAM. To further verify the encapsulation of AHU-1 and Ce6, the UV-vis absorption spectra of AM, CM and CAM were characterized (Fig. 1d). Two main bands at $291 \mathrm{~nm}$ and $372 \mathrm{~nm}$ were observed for AM, and two more bands at $403 \mathrm{~nm}$ and $652 \mathrm{~nm}$ were observed for CM. In view of the UV-vis absorption spectra of CAM (main bands at $285 \mathrm{~nm}, 404 \mathrm{~nm}$ and $659 \mathrm{~nm}$ ) in PBS, it was obvious that the spectra of CAM almost matched the superposition of the spectra of CM and AM in PBS, further proving the successful encapsulation of Ce6 and AHU-1 in the micelles. Then, the transmission electron microscopy (TEM) images of AHU-1 and CAM are shown in Fig. 1b. The images revealed that the diameter of AHU-1 appeared in the range of 2-3 $\mathrm{nm}$ and AHU-1 was successfully encapsulated into CAM, which showed spherical morphologies. The CAM sample had an average particle size of $27.15 \pm 11.30 \mathrm{~nm}$ (Table S1†). The size distributions of BM, CM, AM, and CAM were characterized using DLS, and the average sizes were $20.94 \pm 3.15 \mathrm{~nm}, 22.67 \pm$ $4.32 \mathrm{~nm}, 25.56 \pm 3.01 \mathrm{~nm}$ and $27.46 \pm 4.77 \mathrm{~nm}$, respectively (Fig. 1c and DLS details see Fig. $\mathrm{S} 1 \dagger$ ). Such nanoscale sizes could benefit micelles to preferably accumulate at the tumor site by enhanced permeability and retention (EPR) effect. ${ }^{24}$ The negative zeta-potential $-12.5 \pm 0.68 \mathrm{mV}$ of CAM indicated that CAM can prevent macrophage phagocytosis, non-specific binding with endogenous substances and interaction with plasma proteins. $^{25}$ Then, the stability of CAM against the cell culture medium was further investigated using the diameter changes of CAM (Fig. 1e). The diameter of CAM and BM had a negligible change after $36 \mathrm{~h}$ of incubation, indicating that the micelles could remain stable in the cell culture medium, which provides the prerequisite for cell uptake of CAM and guarantees the stability of AHU-1 during cell experiments. The in vitro releasing profiles of CAM were performed under $\mathrm{pH} 6.5$ and $\mathrm{pH} 7.4$, and more than $50 \%$ of both contents was maintained in the micelles within 10 hours (ESI Fig. S2†).
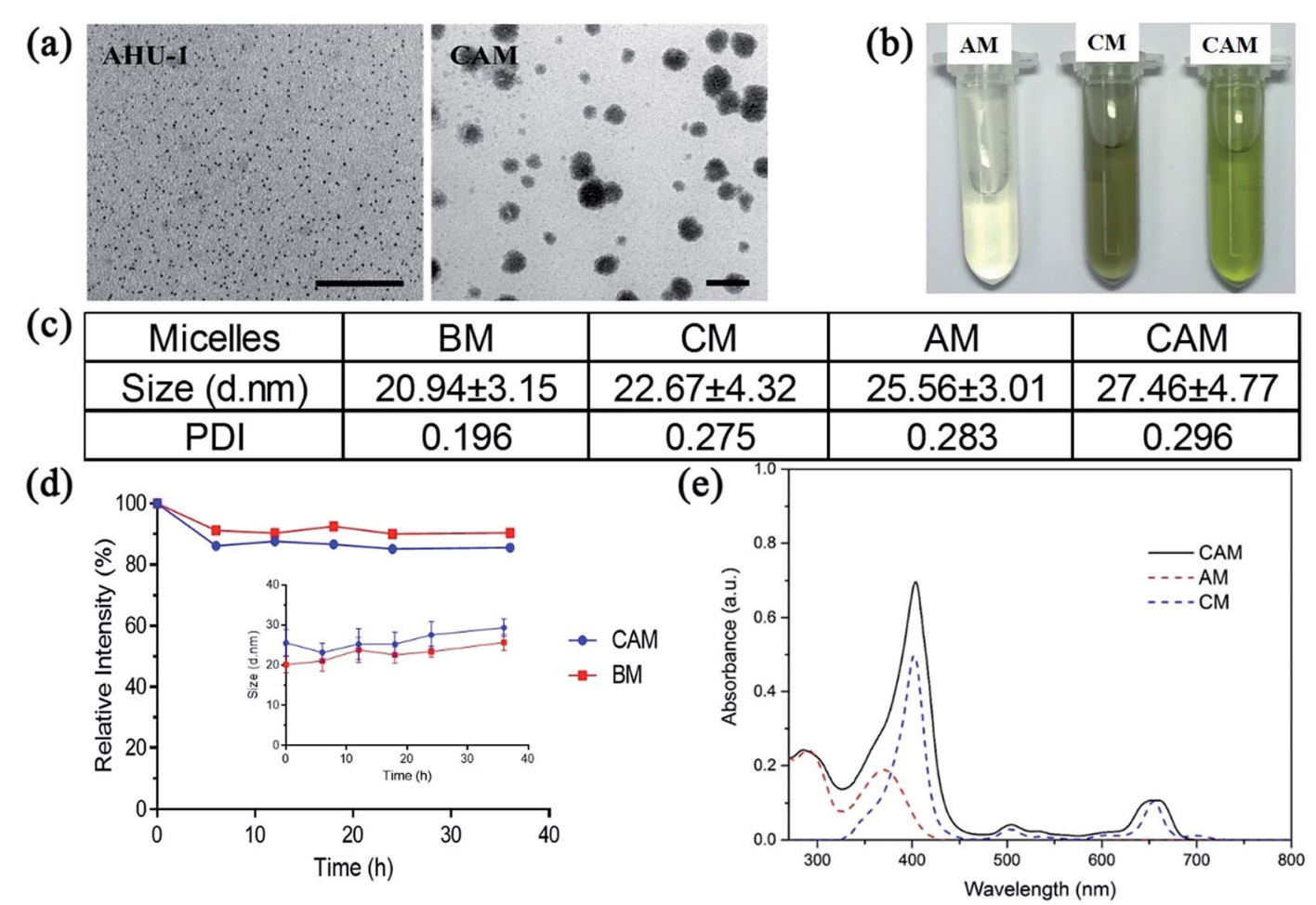

Fig. 1 (a) TEM images of AHU-1 (left) and CAM (right). Scale bars represent $100 \mathrm{~nm}$. (b) Images of AM, CM and CAM solutions. (c) Average sizes of BM, CM, AM and CAM by DLS. (d) The relative intensity and (inset) average sizes of CAM and BM particles in RPMI 1640 medium with $10 \%$ (v/v) fetal bovine serum within $36 \mathrm{~h}$ under $37^{\circ} \mathrm{C}$. (e) UV-vis absorption spectra of $C A M\left(C_{\mathrm{AHU}-1}=5.46 \mu \mathrm{g} \mathrm{mL}^{-1}, C_{\mathrm{Ce} 6}=1.34 \mu \mathrm{g} \mathrm{mL}{ }^{-1}\right), \mathrm{AM}\left(C_{\mathrm{AHU}-1}=5.41 \mu \mathrm{g} \mathrm{mL}{ }^{-1}\right)$ and $\mathrm{CM}\left(\mathrm{C}_{\mathrm{Ce} 6}=1.32 \mu \mathrm{g} \mathrm{mL}{ }^{-1}\right)$. 
(a)

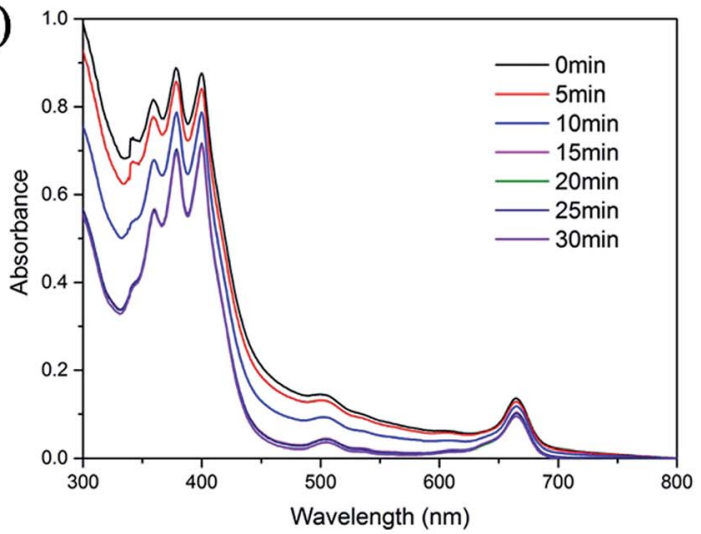

(b)

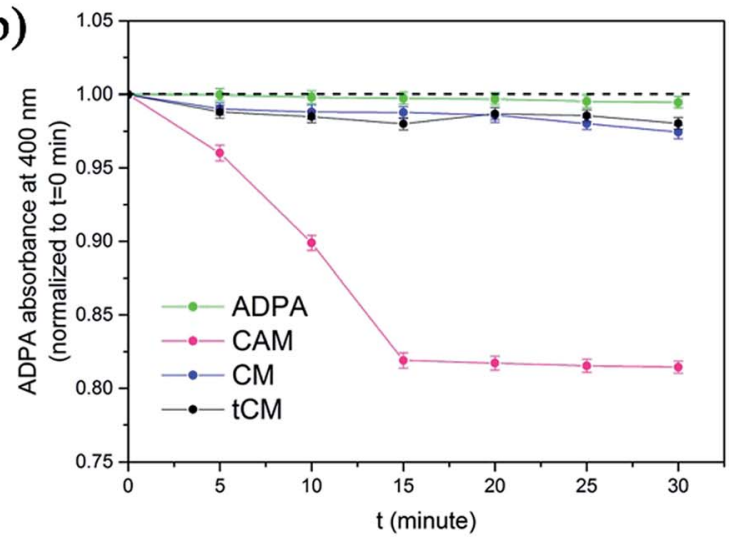

Fig. 2 (a) UV-vis absorption spectra of CAM with ADPA under $808 \mathrm{~nm}$ laser for different illumination times. (b) Singlet oxygen detection of ADPA, CAM + ADPA, CM + ADPA and tCM + ADPA group under irradiation of $808 \mathrm{~nm}$ laser by measuring the absorbance of ADPA at $400 \mathrm{~nm}$.

\subsection{In vitro singlet oxygen $\left({ }^{1} \mathrm{O}_{2}\right)$ generation}

Reactive oxygen species (ROS), particularly singlet oxygen, are generally considered to be the crucial therapeutic element in PDT. A sensitive probe of ROS, 9,10-anthracene-dipropionic acid disodium salt (ADPA), was used to detect the generation of ${ }^{1} \mathrm{O}_{2}$ from CAM by monitoring the absorbance decay at $400 \mathrm{~nm}$. Compared to the CM + ADPA group and the ADPA group, the absorbance of ADPA in CAM decreased along with the time of laser irradiation $(808 \mathrm{~nm})$, which suggested the excitation of Ce6 by NIR laser (Fig. 2a and b). In order to elucidate that Ce6 was indirectly excited via SHG emission instead of two-photon fluorescence from the centrosymmetric stacking organic ligand (tpatpy), the Ce6/tpatpy co-loaded micelles (tCM) were prepared. As shown in Fig. 2b, tCM was incapable of generating ${ }^{1} \mathrm{O}_{2}$, which proved that SHG emission from AHU-1 contributed significantly to the generation of ${ }^{1} \mathrm{O}_{2}$ rather than TPEF during irradiation.

\subsection{In vitro dark cytotoxicity assay}

MTT assays were used to evaluate the cytotoxicity of free tpatpy (in DMSO), free Ce6 (in DMSO), BM, AM, CM and CAM on B16 melanoma cells under dark conditions. DMSO was used as a solvent for the stock solution of tpatpy and Ce6. DMSO was diluted with PBS to reach a 'safe' concentration of $0.1 \%(\mathrm{v} / \mathrm{v})$ in the culture medium. Compared with the free tpatpy group (Fig. 3a), it could be observed that the cell viability was kept above $70 \%$ after $24 \mathrm{~h}$ incubation with BM and AM by dosing up to $50 \mu \mathrm{g} \mathrm{mL} \mathrm{m}^{-1}$ (Fig. 3b). The results indicated that the cytotoxicity was hardly induced by MPEG-PDLLA or the encapsulated SHG materials. Furthermore, in view of the high cytotoxicity of free Ce6, the cell viability of CM was maintained over $40 \%$ even though dosing was up to $20 \mu \mathrm{g} \mathrm{mL} \mathrm{m}^{-1}$, which was much higher than the ordinary cellular PDT concentration $\left(5 \mu \mathrm{g} \mathrm{mL} \mathrm{m}^{-1}\right)$. Above all, it was indicated that these micelle samples had low cytotoxicity and could be further used for cellular uptake study and in vitro PDT.

\subsection{Cellular uptake study}

Cellular uptake of AM and CAM was investigated by confocal laser scanning microscopy (CLSM) in B16 melanoma cells. From Fig. 4, it can be clearly found that a strong green twophoton fluorescence of AHU-1 exists in the cytoplasm under
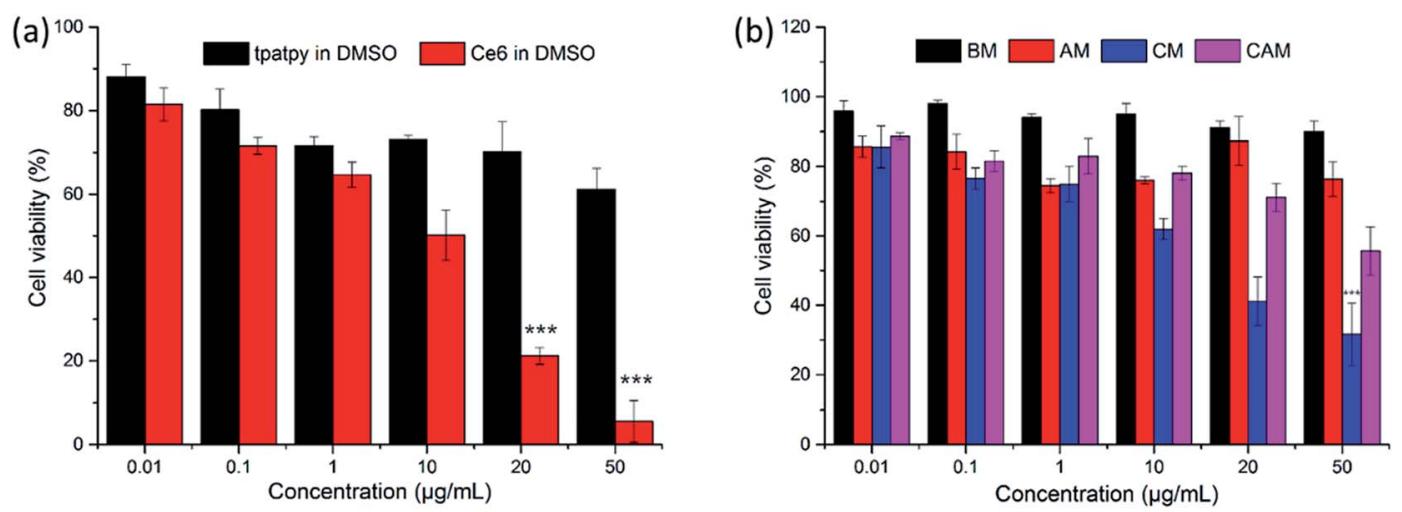

Fig. 3 (a) Viabilities of B16 melanoma cells incubated with free tpatpy (in DMSO) and Ce6 (in DMSO) $* * * P<0.01$ relative to Ce6 at $0.01 \mu \mathrm{g} \mathrm{mL}{ }^{-1}$ by using a one-way ANOVA; (b) viabilities of $\mathrm{B} 16$ melanoma cells incubated with BM $\left(C_{\mathrm{mPEG}-\mathrm{PDLLA}}\right), \mathrm{AM}\left(C_{\mathrm{AHU}-1}\right), \mathrm{CM}\left(C_{\mathrm{Ce} 6}\right), \mathrm{CAM}\left(C_{\mathrm{AHU}-1}, C_{\mathrm{AHU}-1}: C_{\mathrm{Ce} 6}=\right.$ 2 : 1) in the dark. ${ }^{* * *} P<0.01$ relative to $C M$ at polymer concentration of $0.01 \mu \mathrm{g} \mathrm{mL}{ }^{-1}$ by using a one-way ANOVA. 


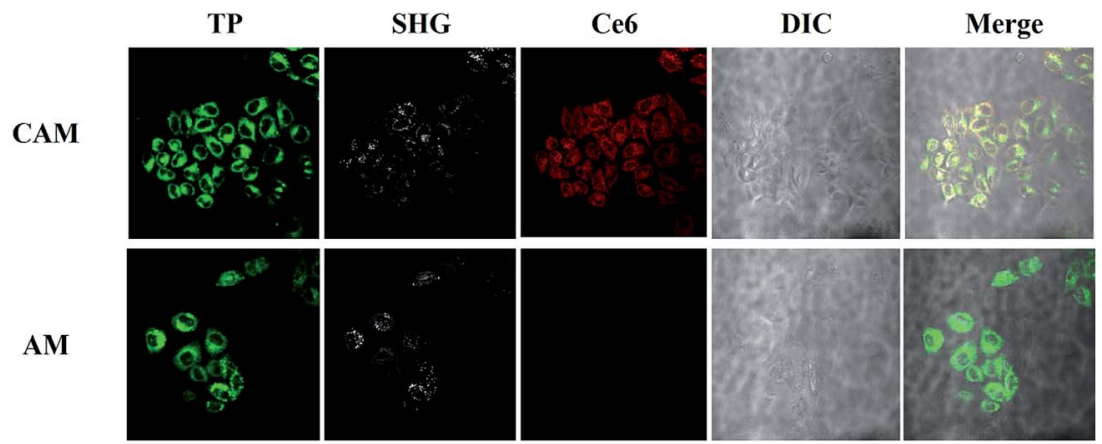

Fig. 4 Living cell one-photon, two-photon and SHG micrographs use CAM and AM. AHU-1 was excited at $760 \mathrm{~nm}$ and $980 \mathrm{~nm}$. Ce6 was excited at $610 \mathrm{~nm}$.

the excitation of $760 \mathrm{~nm}$ laser. Under $980 \mathrm{~nm}$ laser excitation, an intense SHG signal of AHU- 1 could be observed in CAM and AM, which indicated that AHU-1 could remain stable and generate SHG emission. Moreover, red fluorescence of $\mathrm{Ce} 6$ was generated intracellularly. The overlapping of SHG, red and green channels of CAM dosed cells indicated that AHU-1 and Ce6 were

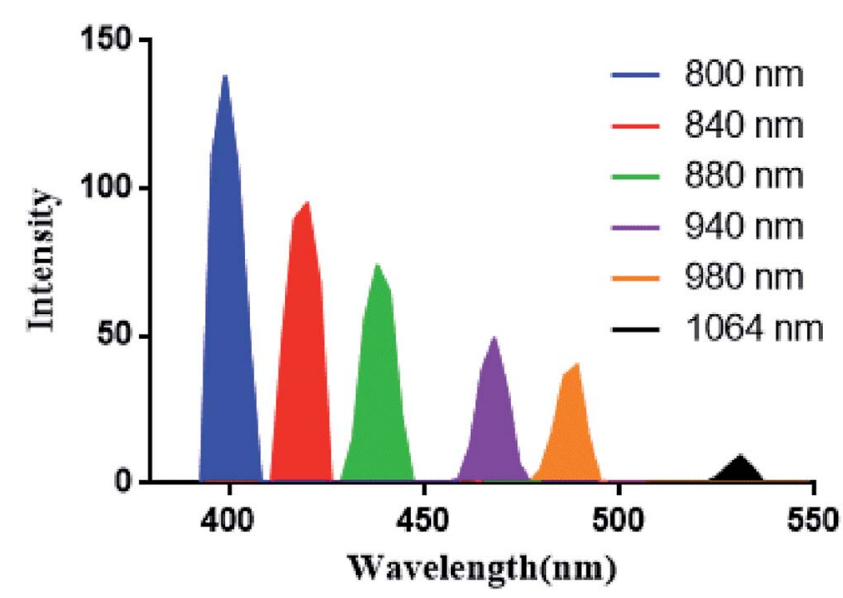

Fig. 5 SHG spectra of AHU-1 extracted from lambda stack imaging of AM treated cells recorded at different incident wavelengths. successfully encapsulated and simultaneously delivered into cells. Apart from the cellular uptake study of micelles, the SHG spectra of AM in the cells were recorded at different incident wavelengths from 800 to $1064 \mathrm{~nm}$ by the lambda $(\lambda)$-stacking technique. AHU-1 showed a remarkable SHG signal at a narrow emission window, which was exactly close to half of the incident wavelengths (Fig. 5). Such an optical property would allow controlling SHG materials elastically exciting any PSs by simply adjusting the incident wavelengths.

\subsection{In vitro PDT effect}

The in vitro PDT efficacy was determined via MTT assays and performed in situ under CLSM. As shown in Fig. 6a, compared to the control group treated with an equivalent light dose, the viability of cells incubated with CAM decreased gradually with the extension of illumination time. More than $60 \%$ of the cancer cells were eliminated after 10 min irradiation, which showed the high efficiency of in vitro PDT upon $808 \mathrm{~nm}$ laser irradiation. Moreover, in situ morphological change of cells in the early stages of PDT was captured under a microscope. As shown in Fig. 6b, several spherical bubbles and unclear cell edges were observed on cell membranes and the nucleus. The morphological changes of cells were associated with cell apoptosis (and/ or necrosis). (a)

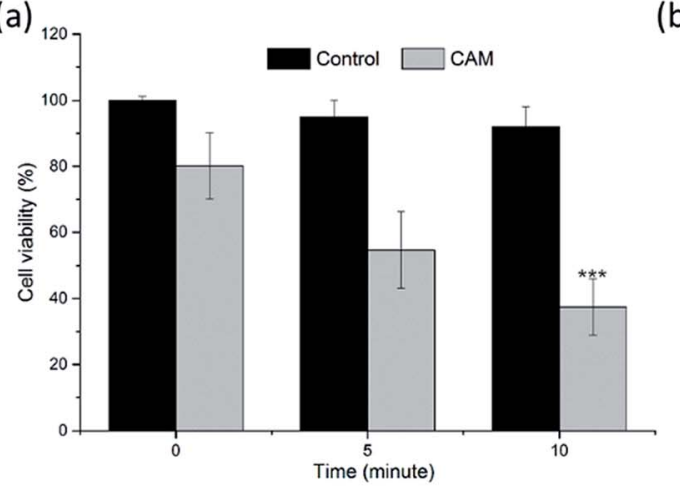

(b)

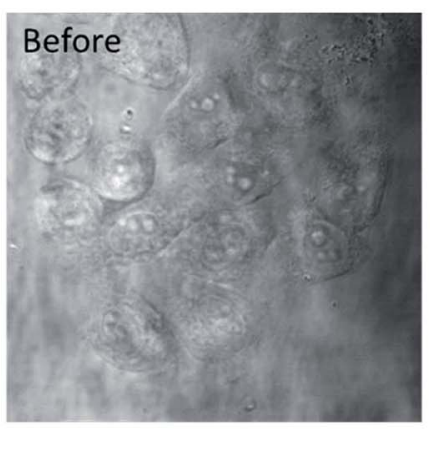

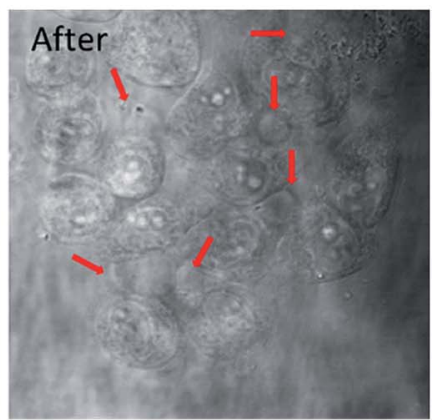

Fig. 6 (a) Viability of B16 melanoma cells incubated with CAM after $808 \mathrm{~nm}$ laser irradiation. ***P<0.01 relative to viability at 0 min by using a one-way ANOVA. (b) Morphological changes of B16 melanoma cells in the early stages after photodynamic therapy. (A video of the cell morphology collapsing is provided in the ESI $\dagger$ ). 


\section{Conclusions}

In summary, we reported the preparation and characterization of co-encapsulation of a high SHG generating coordination polymer (AHU-1) and a photosensitizer (Ce6) into biocompatible mPEG-PDLLA micelles against melanoma cancer by SHGmediated PDT. The application of micelles ensured the stability of AHU-1 and Ce6 in an aqueous environment and CAM produced large amounts of ${ }^{1} \mathrm{O}_{2}$ through SHG-mediated PDT. Then, the live cell imaging and viability assays demonstrated the intracellular co-delivery of both cargoes by CAM and significant PDT efficacy upon NIR laser irritation. Therefore, the SHG-mediated photodynamic therapy provides a novel strategy for the application of nonlinear optical materials in NIR PDT.

\section{Conflicts of interest}

There are no conflicts to declare.

\section{Acknowledgements}

We acknowledge the financial support from the National Natural Science Foundation of China (No. 81503009), the National key research and development program of China (No. 2017YFD0501404), and the Chongqing Science and Technology Commission (No. cstc2015shmszx80018, cstc2016jcyjA0550).

\section{References}

1 P. Agostinis, K. Berg, K. A. Cengel, T. H. Foster, A. W. Girotti, S. O. Gollnick, et al., Photodynamic therapy of cancer: an update, Ca-Cancer J. Clin., 2011, 61, 250-281.

2 S. Mallidi, S. Anbill, A. L. Bulin, G. Obaid, M. Ichikawa and T. Hasan, Beyond the Barriers of Light Penetration: Strategies, Theranostics, 2016, 6, 2458-2487.

3 G. M. F. Calixto, J. Bernegossi, L. M. d. Freitas, C. R. Fontana and M. Chorilli, Nanotechnology-Based Drug Delivery Systems for Photodynamic Therapy of Cancer: A Review, Molecules, 2016, 21, 342-359.

4 B. Liu, C. Li, Z. Cheng, Z. Hou, S. Huang and J. Lin, Functional nanomaterials for near-infrared-triggered cancer therapy, Biomater. Sci., 2016, 4, 890-909.

5 Q. Zou, H. Zhao, Y. Zhao, Y. Fang, D. Chen, J. Ren, X. Wang, Y. Wang, Y. Gu and F. Wu, Effective Two-Photon Excited Photodynamic Therapy of Xenograft Tumors Sensitized by Water-Soluble Bis(arylidene)cycloalkanone Photosensitizers, J. Med. Chem., 2015, 58, 7949-7958.

6 S. Schastak, B. Jean, R. Handzel, G. Kostenich, R. Hermann, U. Sack, A. Orenstein, Y. Wang and P. Wiedemann, Improved pharmacokinetics, biodistribution and necrosis in vivo using a new near infra-red photosensitizer: tetrahydroporphyrin tetratosylat, J. Photochem. Photobiol., B, 2005, 78, 203-213.

7 C. Wang, H. Tao, L. Cheng and Z. Liu, Near-infrared light induced in vivo photodynamic therapy of cancer based on upconversion nanoparticles, Biomaterials, 2011, 32, 61456154.
8 M. Kruka, A. Karotki, M. Drobizhev, V. Kuzmitsky, V. Gael and A. Rebane, Two-photon absorption of tetraphenylporphin free base, J. Lumin., 2003, 105, 45-55.

9 J. Wójcik, J. Peszke, A. Ratuszna, P. Ku and R. Wrzalik, Theoretical investigation of porphyrin-based photosensitizers with enhanced NIR absorption, Phys. Chem. Chem. Phys., 2013, 15, 19651-19658.

10 M. C. DeRosa and R. J. Crutchley, Photosensitized singlet oxygen and its applications, Coord. Chem. Rev., 2002, 233, 351-371.

11 S. Cui, D. Yin, Y. Chen, Y. Di, H. Chen, Y. Ma, S. Achilefu and Y. Gu, In Vivo Targeted Deep-Tissue Photodynamic Therapy Based on Near-Infrared Light Triggered Upconversion Nanoconstruct, ACS Nano, 2013, 7, 676-688.

12 Y. Shen, A. J. Shuhendler, D. Ye, J. Xu and H. Chen, Twophoton excitation nanoparticles for photodynamic therapy, Chem. Soc. Rev., 2016, 45, 6725-6741.

13 W. R. Dichtel, J. M. Serin, C. Edder, J. M. J. Fréchet, M. Matuszewski, L. Tan, T. Y. Ohulchanskyy and P. N. Prasad, Singlet Oxygen Generation via Two-Photon Excited FRET, J. Am. Chem. Soc., 2004, 126, 5380-5381.

14 P. Pantazis and H. B. Gray, Second harmonic generating (SHG) nanoprobes for in vivo imaging, Proc. Natl. Acad. Sci. U. S. A., 2010, 107, 14535-14540.

15 S. Yoshiyagawa, K. Tohda, Y. Umezawa, S. Hashimoto and M. Kawasaki, Optical Ion Sensor Based on SecondHarmonic Generation at the Surface of an IonophoreIncorporated Poly(vinyl chloride) Liquid Membrane, Anal. Sci., 2007, 9, 715-718.

16 P. J. Campagnola, A. C. Millard, M. Terasaki, P. E. Hoppe, C. J. Malone and W. A. Mohler, Three-Dimensional HighResolution Second-Harmonic Generation Imaging of Endogenous Structural Proteins in Biological Tissues, Biophys. J., 2002, 82, 493-508.

17 S. Wang, X. Zhao, J. Qian and S. He, Polyelectrolyte coated BaTiO3 nanoparticles for second harmonic generation imaging-guided photodynamic therapy with improved stability and enhanced cellular uptake, RSC Adv., 2016, 6, 40615-40625.

18 A. V. Kachynski, A. Pliss, A. N. Kuzmin, T. Y. Ohulchanskyy, A. Baev, J. Qu and P. N. Prasad, Photodynamic therapy by in situ nonlinear photon conversion, Nat. Photonics, 2014, 8, 455-461.

19 A. V. Kachynski, A. Pliss, A. N. Kuzmin, T. Y. Ohulchanskyy, A. Baev, J. Qu and P. N. Prasad, Photodynamic therapy by in situ nonlinear photon conversion, Nat. Photonics, 2014, 8, 455-461.

20 B. Gu, A. Pliss, A. N. Kuzmin, A. Baev, T. Y. Ohulchanskyy, J. A. Damasco, K. Yong, S. Wen and P. N. Prasad, In-situ second harmonic generation by cancer cell targeting $\mathrm{ZnO}$ nanocrystals to effect photodynamic action in subcellular space, Biomaterials, 2016, 10478-10486.

21 J. Su, J. Zhang, X. Tian, M. Zhao, T. Song, et al., A series of multifunctional coordination polymers based on terpyridine and zinc halide: second harmonic generation and two-photon absorption properties and intracellular imaging, J. Mater. Chem. B, 2013, 1-6. 
22 Y. Dong and S. S. Feng, Methoxy poly (ethylene glycol)-poly (lactide) (MPEG-PLA) nanoparticles for controlled delivery of anticancer drugs, Biomaterials, 2004, 25, 2843-2849.

23 J. Lu, Y. Huang, W. Zhao, Y. Chen, J. Li, X. Gao, R. Venkataramanan and S. Li, Design and Characterization of PEG-Derivatized Vitamin $\mathrm{E}$ as a Nanomicellar Formulation for Delivery of Paclitaxel, Mol. Pharmaceutics, 2013, 10, 2880-2890.
24 A. K. Iyer, G. Khaled, J. Fang and H. Maeda, Exploiting the enhanced permeability and retention effect for tumor targeting, Drug Discovery Today, 2006, 11, 812-818.

25 P. Aggarwal, J. B. Hall, C. B. Mcleland, M. A. Dobrovolskaia and S. E. Mcneil, Nanoparticle interaction with plasma proteins as it relates to particle biodistribution, biocompatibility and therapeutic efficacy, Adv. Drug Delivery Rev., 2009, 61, 428-437. 